\title{
Tracking drug-related overdoses at the local level: Using Syndromic Surveillance in the CO-NCR
}

\author{
Emery Shekiro*1, Lily Sussman² and Talia Brown² \\ 1Denver Public Health, Denver, CO, USA; 'Boulder County Public Health, Boulder, CO, USA
}

\section{Objective}

In order to better describe local drug-related overdoses, we developed a novel syndromic case definition using discharge diagnosis codes from emergency department data in the Colorado North Central Region (CO-NCR). Secondarily, we used free text fields to understand the use of unspecified diagnosis fields.

\section{Introduction}

The United States is in the midst of a drug crisis; drug-related overdoses are the leading cause of unintentional death in the country. In Colorado the rate of fatal drug overdose increased 68\% from 20022014 (9.7 deaths per 100,000 to 16.3 per 100,000 , respectively) ${ }^{1}$, and non-fatal overdose also increased during this time period $(23 \%$ increase in emergency department visits since 2011) $)^{2}$. The CDC's National Syndromic Surveillance Program (NSSP) provides nearreal time monitoring of emergency department (ED) events across the country, with information uploaded daily on patient demographics, chief complaint for visit, diagnosis codes, triage notes, and more. Colorado North Central Region (CO-NCR) receives data for 4 local public health agencies from 25 hospitals across Adams, Arapahoe, Boulder, Denver, Douglas, and Jefferson Counties.

Access to local syndromic data in near-real time provides valuable information for local public health program planning, policy, and evaluation efforts. However, use of these data also comes with many challenges. For example, we learned from key informant interviews with ED staff in Boulder and Denver counties, about concern with the accuracy and specificity of drug-related diagnosis codes, specifically for opioid-related overdoses.

\section{Methods}

Boulder County Public Health (BCPH) and Denver Public Health (DPH) developed a query in Early Notification of Community Based Epidemics (ESSENCE) using ICD-10-CM codes to identify cases of drug-related overdose [T36-T51] from October 2016 to September 2017. The Case definition included unintentional, self-harm, assault and undetermined poisonings, but did not include cases coded as adverse effects or underdosing of medication. Cases identified in the query were stratified by demographic factors (i.e., gender and age) and substance used in poisoning. The first diagnosis code in the file was considered the primary diagnosis. Chief complaint and triage note fields were examined to further describe unspecified cases and to describe how patients present to emergency departments in the CONCR. We also explored whether detection of drug overdose visits captured by discharge diagnosis data varied by patient sex, age, or county.

\section{Results}

The query identified 2,366 drug-related overdoses in the CONCR. The prevalence of drug overdoses differed across age groups. The detection of drug overdoses was highest among our youth and young adult populations; 16 to 20 year olds $(16.0 \%), 21-25$ year olds (11.4\%), 26-30 year olds (11.4\%). Females comprised $56.1 \%$ of probable general drug overdoses. The majority of primary diagnoses $(31.0 \%)$ included poisonings related to diuretics and other unspecified drugs (T50), narcotics (T40) $(12.6 \%)$, or non-opioid analgesics
(T39) $(7.8 \%)$. For some cases with unspecified drug overdose codes there was additional information about drugs used and narcan administration found in the triage notes and chief complaint fields.

\section{Conclusions}

Syndromic surveillance offers the opportunity to capture drugrelated overdose data in near-real time. We found variation in drugrelated overdose by demographic groups. Unspecified drug overdose codes are extremely common, which likely negatively impacts the quality of drug-specific surveillance. Leveraging chief complaint and triage notes could improve our understanding of factors involved in drug-related overdose with limitations in discharge diagnosis. Chart reviews and access to more fields from the ED electronic health record could improve local drug surveillance.

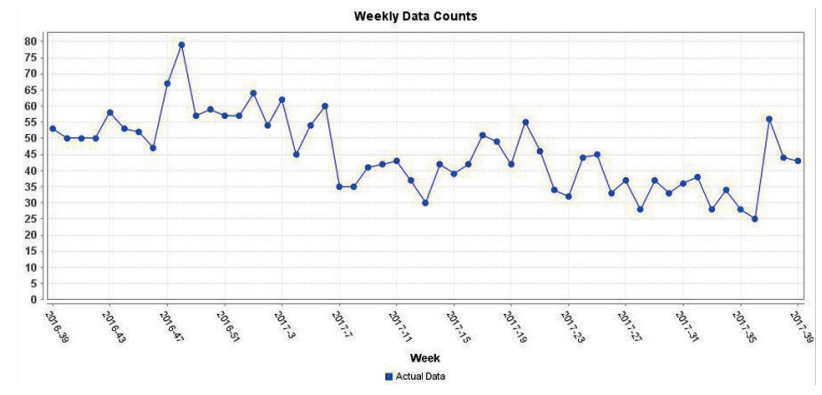

General drug overdose ED visits in the Colorado North Central Region from October 2016 to September 2017 by Week

\section{Keywords}

Syndromic Surveillance; Drug Overdose; ESSENCE

\section{Acknowledgments}

We would like to acknowledge the contributions of Yushiuan Chen, Michele Askenazi, Kathryn DeYoung, Art Davidson, and Christine Billings to the Co-NCR syndromic surveillance system.

\section{References}

1. Colorado Vital Statistics. Death Data. Colorado Department of Public Health and Environment. 2015.

2. Colorado Health and Hospital Association. Hospital Discharge Dataset. 2015.

\section{*Emery Shekiro}

E-mail: Emery.Shekiro@dhha.org 